\title{
Experimental studies on astrophysical reactions at the low- energy RI beam separator CRIB
}

H. Yamaguchi ${ }^{1, *}, S$. Hayakawa $^{1}, N . R . \mathrm{Ma}^{1}, H$. Shimizu $^{1}, K$. Okawa $^{1}, L$. Yang $^{1,2}, D . \mathrm{Kahl}^{1,3,4}$, M. La Cognata ${ }^{5}$, L. Lamia ${ }^{5,6,7}$, K. Abe ${ }^{1}$, O. Beliuskina ${ }^{1,8}$, S.M. Cha ${ }^{9,10}$, K.Y. Chae ${ }^{9}$, S. Cherubini ${ }^{5,6}, P$. Figuera ${ }^{5}, Z . \mathrm{Ge}^{11,8}, M$. Gulino ${ }^{5,12}, J . \mathrm{Hu}^{13}, A$. Inoue $^{14}, N$. Iwasa ${ }^{15}$, A. $\mathrm{Kim}^{16,17}, D . \mathrm{Kim}^{10,16}, G$. Kiss ${ }^{11,18}, S$. Kubono ${ }^{11,13}, M$. La Commara ${ }^{19,20}, M$. Lattuada ${ }^{5,6}$, E.J. Lee ${ }^{9}$, J.Y. Moon ${ }^{21}$, S. Palmerini ${ }^{22,23}$, C. Parascandolo ${ }^{20}$, S.Y. Park ${ }^{16}$, V. H. Phong ${ }^{11,24}$, D. Pierroutsakou ${ }^{20}$, R.G. Pizzone ${ }^{5}$, G.G. Rapisarda ${ }^{5}$, S. Romano ${ }^{5,6,7}$, C. Spitaleri ${ }^{5,6}$,

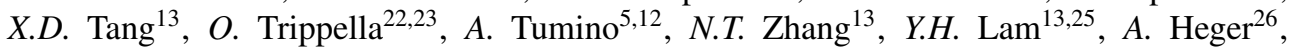
A.M. Jacobs ${ }^{27,28}$, S.W. Xu ${ }^{13}$, S.B. $\mathrm{Ma}^{13}$, L.H. $\mathrm{Ru}^{13}$, E.Q. $\mathrm{Liu}^{13}$, T. $\mathrm{Liu}^{13}$, C.B. Hamill ${ }^{3}$, A. St J. Murphy ${ }^{3}$, J. $\mathrm{Su}^{29}$, X. Fang ${ }^{30}$, M.S. Kwag ${ }^{9}$, N.N. Duy ${ }^{9}$, N.K. Uyen ${ }^{9}$, D.H. Kim ${ }^{9}$, $J$. Liang ${ }^{31}, A$. Psaltis ${ }^{31,32}, M$. Sferrazza ${ }^{33}, Z$. Johnston ${ }^{27,28}$, and $Y . Y . \mathrm{Li}^{34}$

${ }^{1}$ Center for Nuclear Study (CNS), University of Tokyo, Wako, Japan

${ }^{2}$ China Institute of Atomic Energy, Beijing, China

${ }^{3}$ School of Physics and Astronomy, the University of Edinburgh, Edinburgh, UK

${ }^{4}$ Extreme Light Infrastructure - Nuclear Physics, Horia Hulubei National Institute for R\&D in Physics and Nuclear Engineering (IFIN-HH), Bucharest-Măgurele, Romania

${ }^{5}$ Istituto Nazionale di Fisica Nucleare - Laboratori Nazionali del Sud, Catania, Italy

${ }^{6}$ Department of Physics and Astronomy, University of Catania, Catania, Italy

${ }^{7}$ Centro Siciliano di Fisica Nucleare e Struttura della Materia, CSFNSM, Catania, Italy

${ }^{8}$ Department of Physics, University of Jyväskylä, Jyväskylä, Finland

${ }^{9}$ Department of Physics, Sungkyunkwan University, Suwon, Korea

${ }^{10}$ Center for Exotic Nuclear Studies, Institute for Basic Science, Daejeon, Korea

${ }^{11}$ The Institute of Physical and Chemical Research (RIKEN), Wako, Japan

${ }^{12}$ Faculty of Engineering and Architecture, Kore University of Enna, Enna, Italy

${ }^{13}$ Institute of Modern Physics, Chinese Academy of Sciences, Lanzhou, China

${ }^{14}$ Research Center for Nuclear Physics (RCNP), Osaka University, Osaka, Japan

${ }^{15}$ Department of Physics, Tohoku University, Sendai, Japan

${ }^{16}$ Department of Physics, Ewha Womans University, Seoul, Korea

${ }^{17}$ Korea University, Seoul, Korea

${ }^{18}$ Institute for Nuclear Research (Atomki), Debrecen, Hungary

${ }^{19}$ Department of Physics, University of Naples Federico II, Naples, Italy

${ }^{20}$ Istituto Nazionale di Fisica Nucleare - Section of Naples, Naples, Italy

${ }^{21}$ Rare Isotope Science Project, Institute for Basic Science, Daejeon, Korea

${ }^{22}$ Istituto Nazionale di Fisica Nucleare - Section of Perugia, Perugia, Italy

${ }^{23}$ Department of Physics and Geology, University of Perugia, Perugia, Italy

${ }^{24}$ Faculty of Physics, VNU University of Science, Hanoi, Vietnam

${ }^{25}$ School of Nuclear Science and Technology, University of Chinese Academy of Sciences, Beijing, China

${ }^{26}$ School of Physics and Astronomy, Monash University, Victoria, Australia

${ }^{27}$ The Joint Institute for Nuclear Astrophysics, Michigan State University, East Lansing, MI, USA

${ }^{28}$ Department of Physics and Astronomy, Michigan State University, East Lansing, MI, USA

\footnotetext{
*e-mail: yamag@cns.s.u-tokyo.ac.jp
} 
${ }^{29}$ College of Nuclear Science and Technology, Beijing Normal University, Beijing, China

${ }^{30}$ Sino-French Institute of Nuclear Engineering and Technology, Sun Yat-Sen University, Zhuhai, China

${ }^{31}$ Department of Physics \& Astronomy, McMaster University, Ontario, Canada

${ }^{32}$ Technische Universität Darmstadt, Darmstadt, Germany

${ }^{33}$ Département de Physique, Université Libre de Bruxelles, Bruxelles, Belgium

${ }^{34}$ School of Nuclear Science and Technology, Lanzhou University, Lanzhou, China

\begin{abstract}
Experimental studies on astrophysical reactions involving radioactive isotopes (RI) often accompany technical challenges. Studies on such nuclear reactions have been conducted at the low-energy RI beam separator CRIB, operated by Center for Nuclear Study, the University of Tokyo. We discuss two cases of astrophysical reaction studies at CRIB; one is for the ${ }^{7} \mathrm{Be}+n$ reactions which may affect the primordial ${ }^{7} \mathrm{Li}$ abundance in the Big-Bang nucleosynthesis, and the other is for the ${ }^{22} \mathrm{Mg}(\alpha, p)$ reaction relevant in X-ray bursts.
\end{abstract}

\title{
1 Introduction
}

Astrophysical reactions involving radioactive isotopes (RI) often play an important role in explosive stellar environments. Although the RI are seldom seen on the earth due to the finite lifetime, they do exist in stars, and contribute to the evolution and thermal dynamics of stellar objects. Experimental efforts have been made for the studies on such RI-involving reactions. In a normal experimental condition, short-lived RI or neutrons can only be used as the beam, not as the target. However, reaction measurements with RI beams often suffer from the limitation of beam intensities, which are typically as small as $10^{5}$ particles per second (pps) or less, while $>10^{14} \mathrm{pps}$ is available for light-ion beams. This great difference in the beam intensity is fundamental for the feasibility of the measurement.

Here we discuss possible approaches to study RI-involving reactions in spite of the technical limitation of the RI beam, introducing recent representative results from the low-energy RI beam facility CRIB [1-3] of the University of Tokyo. CRIB is an RI beam separator operated by Center for Nuclear Study (CNS), the University of Tokyo, and located at the RIBF facility of RIKEN Nishina Center. CRIB can produce low-energy $(<10 \mathrm{MeV} / \mathrm{u}) \mathrm{RI}$ beams by the in-flight technique, using primary heavy-ion beams accelerated at the AVF cyclotron of RIKEN $(\mathrm{K}=70)$. Most of the RI beams are produced via 2-body reactions such as $(p, n)$, $(d, p)$ and $\left({ }^{3} \mathrm{He}, n\right)$, taking place at an 8-cm-long gas target with a maximum pressure of 760 Torr. A cryogenic target system, in which the target gas can be cooled down to about $90 \mathrm{~K}$, is presently available, and an intense ${ }^{7} \mathrm{Be}$ beam of $2 \times 10^{8} \mathrm{pps}$ was produced with the system [4]. A diagram to show the RI beams ever produced at CRIB is found in [3]. In 2021, a development of ${ }^{6} \mathrm{He}$ beam was carried out, where we successfully produced a ${ }^{6} \mathrm{He}$ beam at $8.0 \mathrm{MeV} / \mathrm{u}$ with an intensity greater than $2 \times 10^{5} \mathrm{pps}$. To further increase the RI beam intensity, a high heat-proof target is under development. Using molybdenum foils as the sealing windows of the gas target, the heat capacity is doubled.

We introduce below two latest works at CRIB [5, 6], in which RI-involving astrophysical reactions were measured at astrophysical energies in indirect ways.

\section{$2{ }^{7} \mathrm{Be}+n$ reaction measurement with the Trojan horse method}

The primordial production of light nuclides is well described with the standard model of Big Bang nucleosynthesis (BBN), however, the ${ }^{7} \mathrm{Li} / \mathrm{H}$ abundance remains overestimated by a factor of 3-4, known as the cosmological lithium problem. We have studied the ${ }^{7} \mathrm{Be}\left(n, p_{0}\right)^{7} \mathrm{Li}$, ${ }^{7} \mathrm{Be}\left(n, p_{1}\right)^{7} \mathrm{Li}^{*}$ and ${ }^{7} \mathrm{Be}(n, \alpha){ }^{4} \mathrm{He}$ reactions, which may affect the primordial ${ }^{7} \mathrm{Li}$ abundance [5]. 
To study the reactions of two unstable particles $\left({ }^{7} \mathrm{Be}\right.$ and $\left.n\right)$, we applied the Trojan horse method (THM, see References in [5,7]), which is an indirect method to study a 2-body reaction via a 3-body reaction measurement under a quasi-free kinematical condition. The ${ }^{7} \mathrm{Be}(n, \alpha){ }^{4} \mathrm{He}$ reaction had been measured with the THM in a previous work [7], and our new work expanded the sensitivity also to the ${ }^{7} \mathrm{Be}\left(n, p_{0}\right)^{7} \mathrm{Li}$ and ${ }^{7} \mathrm{Be}\left(n, p_{1}\right)^{7} \mathrm{Li}^{*}$ reactions, by achieving a sufficient energy resolution to separate the contributions of these two reactions.

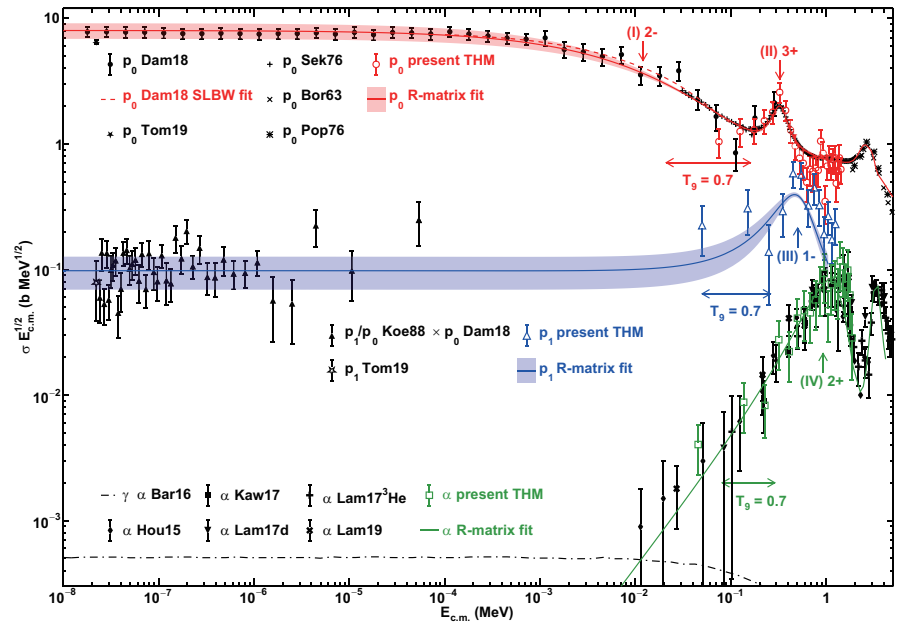

Figure 1. The cross sections multiplied by $\sqrt{E_{\mathrm{cm}}}$ of the ${ }^{7} \mathrm{Be}\left(n, p_{0}\right){ }^{7} \mathrm{Li},{ }^{7} \mathrm{Be}\left(n, p_{1}\right)^{7} \mathrm{Li}{ }^{*}$ and ${ }^{7} \mathrm{Be}(n, \alpha)^{4} \mathrm{He}$ reactions in [5] are shown as open red circles, blue triangles, green squares, respectively. The previous experimental data are also shown for comparison. See [5] for details.

The cross sections multiplied by $\sqrt{E_{\mathrm{cm}}}$ obtained in this work are shown in Fig.1. The ${ }^{7} \mathrm{Be}\left(n, p_{0}\right){ }^{7} \mathrm{Li}$ and ${ }^{7} \mathrm{Be}(n, \alpha){ }^{4} \mathrm{He}$ rates were basically consistent with the previous works, but we assigned a smaller uncertainty on the ${ }^{7} \mathrm{Be}\left(n, p_{0}\right)^{7} \mathrm{Li}$ reaction rate compared to the previous work [8]. The ${ }^{7} \mathrm{Be}\left(n, p_{1}\right)^{7} \mathrm{Li}^{*}$ reaction was measured at the BBN energy for the first time in this work. With the simulation using PRIMAT code, we demonstrated the new reaction rate, including the ${ }^{7} \mathrm{Be}\left(n, p_{1}\right)^{7} \mathrm{Li}^{*}$ contribution, could reduce the primordial ${ }^{7} \mathrm{Li}$ abundance by $\sim 10 \%$.

\section{$3{ }^{25} \mathbf{A l}+p$ elastic resonant scattering for the ${ }^{22} \mathbf{M g}(\alpha, p)$ reaction}

${ }^{22} \operatorname{Mg}(\alpha, p)$ reaction is known to be one of the relevant reactions in X-ray bursts, greatly affecting to its light curve. Recently, the first direct measurement on this reaction has been performed [9], but only at energies much higher than the Gamow energy of X-ray burst, due to the limited intensity ( $900 \mathrm{pps}$ ) of the ${ }^{22} \mathrm{Mg}$ RI beam. The reaction is dominated by several resonances above the $\alpha$-threshold energy, which may imply a simple extrapolation with a statistical-model calculation down to the Gamow energy could be unreliable. Therefore, we studied this reaction by measuring the resonances in the compound ${ }^{26} \mathrm{Si}$ nucleus with resonant scattering of ${ }^{25} \mathrm{Al}+p$.

The measurement was performed at CRIB with an ${ }^{25} \mathrm{Al}$ RI beam at an intensity of $2 \times 10^{5}$ pps with essentially the same method as previous experiments [10, 11]. Fig. 2 shows the obtained excitation functions of the elastic and inelastic scatterings. We obtained resonance parameters just above the $\alpha$ particle threshold, as indicated in the figure, with an R-matrix analysis. The parameters were employed in a new X-ray burst model calculation, and we 
obtained a better agreement with the observed light curves of GS 1826-24 clocked burster and SAX J1808:4-3658 PRE burster compared to the previous works.

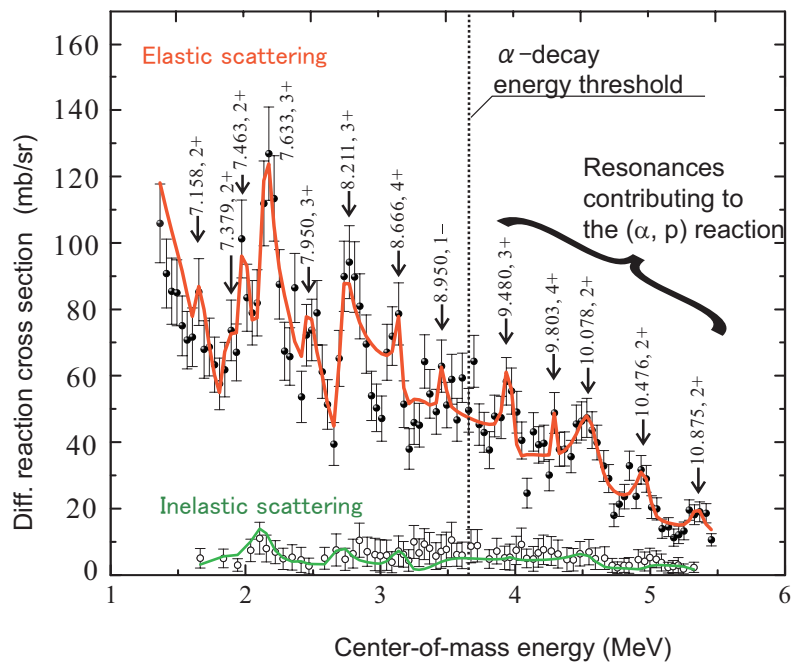

Figure 2. Excitation function of ${ }^{25} \mathrm{Al}+\alpha$ scatterings with an R-matrix analysis. See [6] for details.

\section{Summary}

Astrophysical reactions involving RI are often difficult to study experimentally, mainly due to the limitation of the present RI beam technique. In the above two example, we demonstrated that such astrophysical reactions can be studied even with a low-intensity RI beam, by employing an indirect method (THM) or studying resonance parameters.

The experiments were performed at RI Beam Factory operated by RIKEN Nishina Center and CNS, the University of Tokyo. This work was partly supported by JSPS KAKENHI (No. 18H01218, and 19K03883) from the Ministry of Education, Culture, Sports, Science and Technology (MEXT) of Japan.

\section{References}

[1] S. Kubono, Y. Yanagisawa et al., Eur. Phys. J. A13, 217 (2002)

[2] Y. Yanagisawa, S. Kubono et al., Nucl. Instrum. Meth. Phys. Res., Sect. A 539, 74 (2005)

[3] H. Yamaguchi, D. Kahl et al., Nucl. Phys. News 30, 21 (2020)

[4] H. Yamaguchi, Y. Wakabayashi et al., Nucl. Instrum. Meth. Phys. Res., Sect. A 589, 150 (2008)

[5] S. Hayakawa, M.L. Cognata et al., 915, L13 (2021)

[6] J. Hu, H. Yamaguchi et al., Phys. Rev. Lett. 127, 172701 (2021)

[7] L. Lamia, M. Mazzocco et al., ApJ 879, 23 (2019)

[8] L. Damone, M. Barbagallo et al., PhRvL 121, 042701 (2018)

[9] J.S. Randhawa, Y. Ayyad et al., Phys. Rev. Lett. 125, 202701 (2020)

[10] H. Yamaguchi, Y. Wakabayashi et al., Phys. Lett. B 672, 230 (2009)

[11] J.J. He, L.Y. Zhang et al., Phys. Rev. C 88, 012801 (2013) 